\title{
TWO REMARKS ON A. GLEASON'S FACTORIZATION THEOREM
}

\author{
BY GIOVANNI VIDOSSICH
}

Communicated by Leonard Gillman, August 8, 1969

The theorem of A. Gleason [2, vii.23] asserts that every continuous map $f$ from an open subset $U$ of a product $X$ of separable topological spaces into a Hausdorff space $Y$ whose points are $G_{\delta}$-sets has the form $\left.g \circ \pi\right|_{U}$, where $\pi$ is a countable projection of $X$ and $g: \pi(U) \rightarrow Y$ is continuous. A natural question is to find what other "pleasant" subsets $U$ of $X$ have the above factorization property. The most plausible ones are compact subsets: for, if $U \subseteq X$ is compact and $f=\left.g \circ \pi\right|_{U}$ with $f$ continuous, then $g$ must be continuous since $\left.\pi\right|_{U}$ is a closed map (being continuous on a compact space).

The first part of this note rejects this conjecture by giving an example of a compact subset of a product of copies of the unit interval, without the factorization property. In the second part, it is proved that the factorization $f=\left.g \circ \pi\right|_{U}$ always holds whenever $f$ is uniformly continuous and the range metric. This result implies an open mapping theorem for continuous linear mappings on products of Fréchet spaces.

1. The example. Let $Z$ be a compact Hausdorff space which is first countable but not metrizable. Such a space exists by $[1, \S 2$, Exercise $13]$. Since $Z$ is completely regular, $Z$ is homeomorphic to a compact subset $U$ of a product $X$ of copies of $[0,1]$. Let $f: U \rightarrow U$ be the identity. Assume that $f=\left.g \circ \pi\right|_{U}$, with $\pi$ a countable projection and $g: \pi(U) \rightarrow U$ continuous, and argue for a contradiction. Since countable products of separable metric spaces are separable metric, $\pi(U)$ is separable metric. Hence $U$ is a continuous image of a separable metric space. But a cosmic metric space is metrizable whenever it is compact by $[3$, p. $994,(C)$ for cosmic spaces]. This contradicts the assumptions on $Z$.

2. A factorization theorem. The above example shows that the following result does not hold longer when $Y$ is not metrizable.

THEOREM. If $Z$ is any subset of a product of arbitrary uniform spaces

AMS Subject Classifications. Primary 5425, 5460; Secondary 5440.

Key Words and Phrases. Products of uniform spaces, Banach spaces, countable projections, factorizations upon countable projections, open mapping theorem, products of Banach spaces. 
$X_{\alpha}(\alpha \in A)$ into a metric space $Y$, then every uniformly continuous $f: Z \rightarrow Y$ has the form $\left.g \circ \pi\right|_{z}$ with $\pi$ a countable projection and $g$ uniformly continuous.

Proof. By the uniform continuity of $f$, for each integer $n \geqq 1$ there are a finite subset $A_{n} \subseteq A$ and uniform covers $\varkappa_{\alpha}$ of $X_{\alpha}\left(\alpha \in A_{n}\right)$ such that

$$
d(f(x), f(y)) \leqq 1 / n
$$

whenever $x, y \in Z$ have the coordinates corresponding to $\alpha \in A_{n}$ near of order $\varkappa_{\alpha}$. Put $C=\cup_{n=1}^{\infty} A_{n}$ and $\pi$ the countable projection $\left(x_{\alpha}\right)_{\alpha \in A}$ $\rightarrow\left(x_{\alpha}\right)_{\alpha \in c}$. For every $x \in \pi(Z)$, let $z_{x}$ be a point of $Z \cap \pi^{-1}(x)$. Define $g: \pi(Z) \rightarrow Y$ by $x \rightarrow f\left(z_{x}\right)$. If $z^{\prime}, z^{\prime \prime} \in Z$ have the same image by $\pi$, then $d\left(f\left(z^{\prime}\right), f\left(z^{\prime \prime}\right)\right) \leqq 1 / n$ for all $n \geqq 1$ (since $C \supseteq A_{n}$ ), which implies $d\left(f\left(z^{\prime}\right), f\left(z^{\prime \prime}\right)\right)=0$, i.e. $f\left(z^{\prime}\right)=f\left(z^{\prime \prime}\right)$. This means that $g$ is well defined. From the definition it follows $f=\left.g \circ \pi\right|_{z}$. The equality $f=\left.g \circ \pi\right|_{z}$ means that two points of $Z$ have the same image by $f$ whenever they have the same coordinates for $\alpha \in C$. By this and $C \supseteq A_{n}(n \geqq 1), g$ is uniformly continuous. Q.E.D.

Corollary. Let $X_{\alpha}(\alpha \in A), Y$ be arbitrary complete metrizable topological vector spaces. Then every continuous linear map $f$ from $\prod_{\alpha \in A} X_{\alpha}$ onto $Y$ is open.

Proof. Since a continuous linear map is uniformly continuous in the standard uniformities of topological vector spaces, the above theorem implies that $f=g \circ \pi$, with $\pi$ a countable projection and $g$ uniformly continuous. Since $\pi$ and $f$ are linear, $g$ is linear. Since a countable product of complete metric spaces is complete metric, $g$ is open by Banach homomorphism theorem. Since $\pi$ is open, $f$ must be also. Q.E.D.

\section{REFERENCES}

1. N. Bourbaki, Topologie gênérale. Livre III: Chapitre 9: Utilisation des nombres réels en topologie gênérale, Actualités Sci. Indust., no. 1045, Hermann, Paris, 1958. MR $30 \# 3439$.

2. J. R. Isbell, Uniform spaces, Math. Surveys, no. 12, Amer. Math. Soc., Providence, R.I., 1964. MR $30 \# 561$.

3. E. Michael, $\aleph_{0}$-spaces, J. Math. Mech. 15 (1966), 983-1002. MR 34 \#6723.

University of Pisa, Italy 